\title{
Anisotropic Universe Models with Positive Cosmological Constant
}

\author{
Paulo Aguiar \\ Faculdade de Ciências da Economia e da Empresa, Universidade Lusíada - Norte, Rua Dr. Lopo de Carvalho, 4369-006 Porto, Portugal
}

Copyright (C)2016 by authors, all rights reserved. Authors agree that this article remains permanently

open access under the terms of the Creative Commons Attribution License 4.0 International License

\begin{abstract}
Following the recognition of a positive value for the vacuum energy density and the realization that a simple Kantowski-Sachs model might fit the classical tests of cosmology, we study the qualitative behavior of three anisotropic and homogeneous models: Kantowski-Sachs, Bianchi type-I and Bianchi type-III universes, with dust and a cosmological constant, in order to find out which are physically permitted. We find that these models undergo isotropization up to the point that the observations will not be able to distinguish between them and the standard model, except for the Kantowski-Sachs model $\left(\Omega_{k_{0}}<0\right)$ and for the Bianchi type-III $\left(\Omega_{k_{0}}>0\right)$ with $\Omega_{\Lambda_{0}}$ smaller than some critical value $\Omega_{\Lambda_{M}}$. Even if one imposes that the Universe should be nearly isotropic since the last scattering epoch $(z \approx 1000)$, meaning that the Universe should have approximately the same Hubble parameter in all directions (considering the COBE 4-Year data), there is still a large range for the matter density parameter compatible with Kantowsky-Sachs and Bianchi type-III if $\left|\Omega_{0}+\Omega_{\Lambda_{0}}-1\right| \leq \delta$, for a very small $\delta$. The Bianchi type-I model becomes exactly isotropic owing to our restrictions and we have $\Omega_{0}+\Omega_{\Lambda_{0}}=1$ in this case. Of course, all these models approach locally an exponential expanding state provided the cosmological constant $\Omega_{\Lambda}>\Omega_{\Lambda_{M}}$.
\end{abstract}

Keywords Cosmological Constant, KantowskySachs Model, Bianchi Models, Vacuum Energy Density, Isotropization, Hubble Parameter

\section{Introduction}

Over the last decade, the issue of whether or not there is a nonzero value for the vacuum energy density, or cosmological constant, has been raised quite often. Indeed, the possibility of a nonzero cosmological constant $\Lambda$ has been entertained several times in the past for theoretical and observational reasons (the early history of $\Lambda$ as a parameter in General Relativity has been reviewed by [1], [2], and [3]). Recent supernovae results [4], [5] strongly support a positive and possibly quite large cosmological constant. Even taking the Hubble constant to be in the range $60-75 \mathrm{~km} / \mathrm{s} / \mathrm{Mpc}$ it is possible to show [6] that the standard model of flat space with vanishing cosmological constant is ruled out. In a very nice review [7] it is argued that postulating an $\Omega_{\Lambda}$-dominated model seems to solve a lot of problems at once. And again, in a quite recent review on the physics and cosmology of the cosmological constant, it is added that "recent years have provided the best evidence yet that this elusive quantity does play an important dynamical role in the universe" [8].

On the other hand, if the classical tests of cosmology are applied to a simple Kantowski-Sachs metric and the results compared with those obtained for the standard model, the observations will not be able to distinguish between these models if the Hubble parameters along the orthogonal directions are assumed to be approximately equal [9]. Indeed, as [10] points out, the number of cosmological solutions which demonstrate exact isotropy well after the big bang origin of the Universe is a small fraction of the set of allowable solutions of the cosmological equations. It is therefore prudent to take seriously the possibility that the Universe is expanding anisotropically. Note also that some shear free anisotropic models display a FLRW-like behavior, as it is shown in [11].

\section{The Global Behavior of the $\Lambda \neq$ 0 Models}

Taking all this into consideration, we discuss the behavior of some homogeneous but anisotropic models with axial symmetry, filled with a pressureless perfect fluid (dust) and a non vanishing cosmological constant. For this, we will restrict our study to the the metric forms

$$
d s^{2}=-c^{2} d t^{2}+a^{2}(t) d r^{2}+b^{2}(t)\left(d \theta^{2}+f_{k}^{2}(\theta) d \phi^{2}\right),
$$

with the two scale factors $a(t)$ and $b(t) ; k$ is the curvature index of the 2-dimensional surface $d \theta^{2}+f_{k}^{2}(\theta) d \phi^{2}$ and can take the values $+1,0,-1$ implying $f_{k}(\theta)$ equal $\sin (\theta), \theta, \sinh (\theta)$, respectively, giving the following three 
different metrics: Kantowski-Sachs, Bianchi type-I, and Bianchi type-III [12], [13].

Einstein equations for the metric (1), for which the matter content is in the form of a perfect fluid and a cosmological term, $\Lambda$, are then as follows [12], [13]:

$$
\begin{gathered}
2 \frac{\dot{a}}{a} \frac{\dot{b}}{b}+\frac{\dot{b}^{2}}{b^{2}}+\frac{k c^{2}}{b^{2}}=8 \pi G \rho+\Lambda c^{2}, \\
2 \frac{\ddot{b}}{b}+\frac{\dot{b}^{2}}{b^{2}}+\frac{k c^{2}}{b^{2}}=-8 \pi G \frac{p}{c}+\Lambda c^{2}, \\
\frac{\ddot{a}}{a}+\frac{\ddot{b}}{b}+\frac{\dot{a}}{a} \frac{\dot{b}}{b}=-8 \pi G \frac{p}{c}+\Lambda c^{2},
\end{gathered}
$$

where $\rho$ is the matter density and $p$ is the (isotropic) pressure of the fluid. Here $G$ is the Newton's gravitational constant and $c$ is the speed of light. If we consider a vanishing pressure $(p=0)$, which is compatible with the present conditions for the Universe, the last two equations take the form

$$
\begin{gathered}
2 \frac{\ddot{b}}{b}+\frac{\dot{b}^{2}}{b^{2}}+\frac{k c^{2}}{b^{2}}=\Lambda c^{2}, \\
\frac{\ddot{a}}{a}+\frac{\ddot{b}}{b}+\frac{\dot{a}}{a} \frac{\dot{b}}{b}=\Lambda c^{2},
\end{gathered}
$$

and equation (5) can easily be integrated to give

$$
\frac{\dot{b}^{2}}{b^{2}}=\frac{M_{1}}{b^{3}}-\frac{k c^{2}}{b^{2}}+\frac{\Lambda}{3} c^{2},
$$

where $M_{1}$ is a constant of integration.

The Hubble parameters corresponding to the scale factors $a(t)$ and $b(t)$ are defined by

$$
H_{a} \equiv \dot{a} / a \quad \text { and } \quad H_{b} \equiv \dot{b} / b .
$$

Using them to introduce the following dimensionless parameters, in analogy with which it is usually done in the Friedmann-Lemaître-Robertson-Walker (FLRW) universes, let us define

$$
\begin{gathered}
\frac{M_{1}}{b^{3} H_{b}^{2}} \equiv \Omega_{M}, \\
-\frac{k c^{2}}{b^{2} H_{b}^{2}} \equiv \Omega_{k}
\end{gathered}
$$

and

$$
\frac{\Lambda c^{2}}{3 H_{b}^{2}} \equiv \Omega_{\Lambda}
$$

The conservation equation (7) can now be rewritten as

$$
\Omega_{M}+\Omega_{k}+\Omega_{\Lambda}=1
$$

Now defining the dimensionless variable $y=b / b_{0}$ where $b_{0}=b\left(t_{0}\right)$ is the angular scale factor for the present age of the Universe, and using equation (11) (taken for $t=t_{0}$ ), one may rewrite equation (7) as

$$
\dot{y}= \pm H_{b_{0}} \sqrt{\Omega_{M_{0}}\left(\frac{1}{y}-1\right)+\Omega_{\Lambda_{0}}\left(y^{2}-1\right)+1},
$$

where the density parameters defined previously and $H_{b}$ with the zero subscript, denote as before these quantities at the present time $t_{0}$. In this way, the number of independent parameters have been reduced. Substituting equation (7) into equation (2) gives

$$
\dot{a}=\frac{M_{\rho}-M_{1} \frac{a}{b}+\frac{2}{3} \Lambda c^{2} a b^{2}}{2 \sqrt{M_{1} b-k c^{2} b^{2}+\frac{\Lambda}{3} c^{2} b^{4}}},
$$

where $M_{\rho}$ is a constant proportional to the matter in the Universe,

$$
M_{\rho}=8 \pi G \rho a b^{2} .
$$

Using the procedure above, equation (13) can be rewritten in the form

$$
\Omega_{\rho}-\Omega_{M}+2 \Omega_{\Lambda}=2 \frac{H_{a}}{H_{b}}
$$

where

$$
\Omega_{\rho}=\frac{M_{\rho}}{a b^{2} H_{b}^{2}} .
$$

From equation (2) one may define a matter density parameter. For this, we introduce the notion of mean Hubble factor $H$ such that $3 H=H_{a}+2 H_{b}$. Also, for these models, the shear scalar $\sigma$ [13] is given by

$$
\sigma=\frac{1}{\sqrt{3}}\left(H_{a}-H_{b}\right)
$$

Thus, equation (2) may be rewritten [12] as

$$
3 H^{2}+\frac{k c^{2}}{b^{2}}=8 \pi G \rho+\sigma^{2}+\Lambda c^{2} .
$$

As in FLRW we call critical matter density $\rho_{c}$ when $k=0$ and $\Lambda=0$

$$
\rho_{c}=\frac{3 H^{2}-\sigma^{2}}{8 \pi G} .
$$

The matter density is generally defined as $\Omega=\rho / \rho_{c}$, then

$$
\Omega=\frac{8 \pi G \rho}{3 H^{2}-\sigma^{2}} \equiv \frac{8 \pi G \rho}{2 H_{a} H_{b}+H_{b}^{2}},
$$

just like in FLRW models, and such that $\Omega=1$ when $k=0$ and $\Lambda=0$, and which is related to $\Omega_{\rho}$ by

$$
\Omega=\frac{\Omega_{\rho}}{1+2 \frac{H_{a}}{H_{b}}} .
$$

Although $\Omega_{M}$ is not the matter density parameter, it performs the same important role. We emphasize the fact that if for one particular time $H_{a}=H_{b}$ and $\Omega_{\Lambda}=$ 1 , then, by equations (11), (15) and (21), $3 \Omega=\Omega_{\rho}=$ $\Omega_{M}=-\Omega_{k} ;$ and if $0<\Omega_{\Lambda} \ll 1$ and $\Omega_{M}=1$, then, $-\Omega_{k}=\Omega_{\Lambda}$ and $\Omega \simeq 1$.

Introducing another dimensionless variable $x=a / a_{0}$, equation (13) takes the form

$$
\dot{x}=H_{b_{0}} \frac{\frac{\Omega_{M_{0}}}{2}\left(1-\frac{x}{y}\right)+\Omega_{\Lambda_{0}}\left(-1+x y^{2}\right)+\frac{H_{a_{0}}}{H_{b_{0}}}}{y \sqrt{\Omega_{M_{0}}\left(\frac{1}{y}-1\right)+\Omega_{\Lambda_{0}}\left(y^{2}-1\right)+1}},
$$

and its number of independent parameters was also reduced, now at the expense of equation (15) taken for the present time $t=t_{0}$.

Now, we want to find the time dependence of $b(t)$ in a qualitative way, starting from equation (12). Since the 
model universe will be defined only where $\dot{y}^{2} \geq 0$, as was previously done by [14] for FLRW models, the problem is reduced to find out the zeros of $\dot{y}$, with $y \neq 0$.

There are two $\Omega_{\Lambda}$ values which characterize two zones of distinct behavior for the scale factor $b$. Starting with condition $\dot{y}=0$ one may obtain

$$
\Omega_{\Lambda_{0}}=\frac{\left(\Omega_{M_{0}}-1\right) y-\Omega_{M_{0}}}{y^{3}-y}
$$

If we consider $\Omega_{\Lambda_{0}}=\Omega_{\Lambda_{0}}(y)$, as a function of $y$, then this function presents a relative minimum and a maximum, that we will denote by $\Omega_{\Lambda_{c}}$ and $\Omega_{\Lambda_{M}}$, respectively. The relative minimum depends on $\Omega_{M_{0}}$ in the following way [14]: For $\Omega_{M_{0}}<1 / 2$ we have

$$
\begin{aligned}
\Omega_{\Lambda_{c}}= & \frac{3 \Omega_{M_{0}}}{2}\left\{\left[\sqrt{\frac{\left(\Omega_{M_{0}}-1\right)^{2}}{\Omega_{M_{0}}^{2}}-1}+\frac{1-\Omega_{M_{0}}}{\Omega_{M_{0}}}\right]^{1 / 3}+\right. \\
& \left.\frac{1}{\left[\sqrt{\left(\Omega_{M_{0}}-1\right)^{2} / \Omega_{M_{0}}^{2}-1}+\left(1-\Omega_{M_{0}}\right) / \Omega_{M_{0}}\right]^{1 / 3}}\right\} \\
& -\left(\Omega_{M_{0}}-1\right),
\end{aligned}
$$

for $\Omega_{M_{0}} \geq 1 / 2$ the expression is

$$
\Omega_{\Lambda_{c}}=-3 \Omega_{M_{0}} \cos \left(\frac{\theta+2 \pi}{3}\right)-\left(\Omega_{M_{0}}-1\right) .
$$

The relative maximum is done by

$$
\Omega_{\Lambda_{M}}=-3 \Omega_{M_{0}} \cos \left(\frac{\theta+4 \pi}{3}\right)-\left(\Omega_{M_{0}}-1\right),
$$

where $\theta=\cos ^{-1}\left(\frac{\Omega_{M_{0}}-1}{\Omega_{M_{0}}}\right)$. These expressions are limiting zones of the $\left(\Omega_{\Lambda_{0}}, \Omega_{M_{0}}\right)$ plane, where $\dot{y}=0$ has three or one solutions (for details see [14]). The $\Omega_{\Lambda_{M}}$ expression is also defined for $\Omega_{M_{0}}>1 / 2$, but it has the meaning of a maximum only for $\Omega_{M_{0}}>1$. The $\Omega_{\Lambda_{0}}$ less or equal to $\Omega_{\Lambda_{M}}$ imposes the recollapse of scale factor $b$, while greater values produces inflexional behaviors for $b$. The $\Omega_{\Lambda_{0}}$ values greater or equal to $\Omega_{\Lambda_{c}}$ are physically "forbidden" because they don't reproduce the present Universe (see [14]). Obviously, $\Omega_{\Lambda_{M}}<\Omega_{\Lambda_{c}}$ always.

Although we are considering anisotropic models, the equation (12) for $\dot{y}$ as a function of $\Omega_{M_{0}}$ is mathematically the same as equation (2) obtained by [14] for the homogeneous and isotropic FLRW models. From equations (12) and (22) one obtains the differential equation

$$
\frac{d x}{d y}=\frac{\frac{\Omega_{M_{0}}}{2}\left(1-\frac{x}{y}\right)+\Omega_{\Lambda_{0}}\left(-1+x y^{2}\right)+\frac{H_{a_{0}}}{H_{b_{0}}}}{\Omega_{M_{0}}(1-y)+\Omega_{\Lambda_{0}}\left(y^{3}-y\right)+y} .
$$

This equation automatically complies with the two conservation equations (11) and (15) evaluated at $t_{0}$. There are some particular values of the parameters $\left(\Omega_{M_{0}}, \Omega_{\Lambda_{0}}\right)$ for which this equation has exact solutions. However, for the majority of the values of the parameters, the solution has only been obtained by numerical integration.

We may admit that at a certain moment of time, which we can take as the present time $t_{0}$, the Hubble parameters along the orthogonal directions may be assumed to be approximately equal, $H_{a} \simeq H_{b}$, even though we started with an anisotropic geometry. This hypothesis has been considered in [9] for the case of a Kantowski-Sachs (KS) model. From this study was derived the conclusion that the classical tests of cosmology are not at present sufficiently accurate to distinguish between a FLRW model and the KS defined in that paper, with $\left(H_{a_{0}} \simeq H_{b_{0}}\right)$, except for small values of $b_{0}$.

Taking $H_{a_{0}}=H_{b_{0}}$ for simplicity, one can then integrate equation (27) and find three different solutions, one for each $k$ value. Figure 1 and Figure 2 shows the three kinds of behaviors as a result of integration.

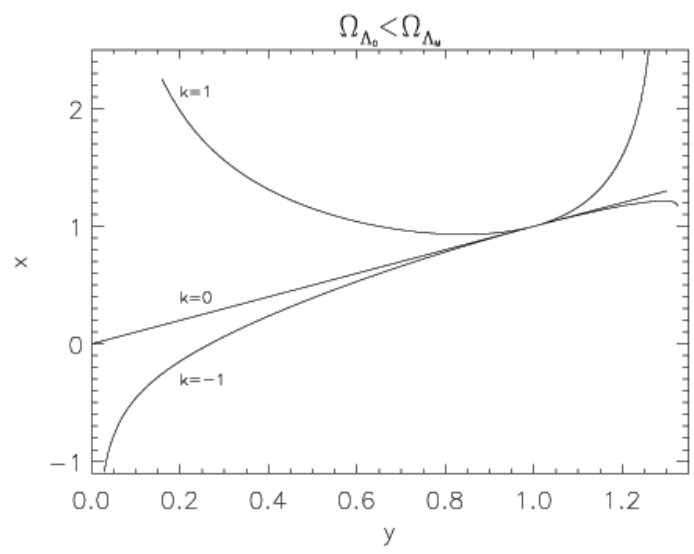

Figure 1. Scale factors relation, that is, the $x y$ dependence for the three models Kantowski-Sachs $(k=1)$, Bianchi type-I $(k=0)$ and Bianchi type-III $(k=-1)$. We show the behaviour of $x(y)$ when $\Omega_{\Lambda_{0}}<\Omega_{\Lambda_{M}}$. Concretely we have for KantowskiSachs $\Omega_{M_{0}}=9$ and $\Omega_{\Lambda_{0}}=1.5$; for Bainchi type-I $\Omega_{M_{0}}=2$ and $\Omega_{\Lambda_{0}}=-1$; for Bainchi type-III $\Omega_{M_{0}}=1$ and $\Omega_{\Lambda_{0}}=-1$.

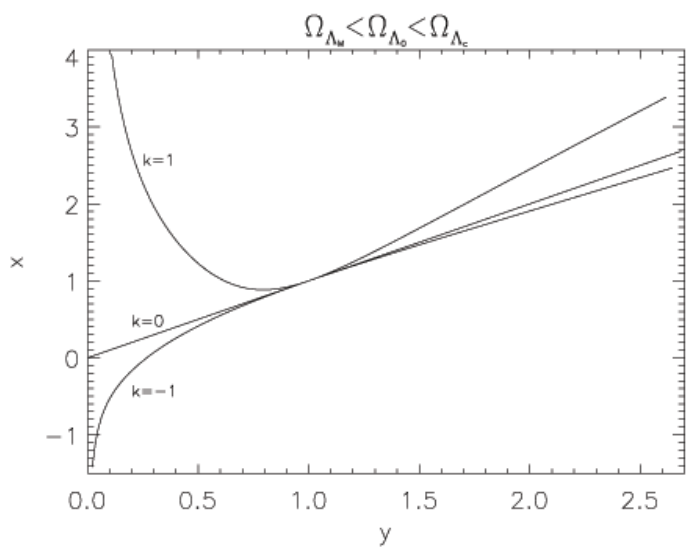

Figure 2. Scale factors relation, that is, the $x y$ dependence for the three models Kantowski-Sachs $(k=1)$, Bianchi type-I $(k=0)$ and Bianchi type-III $(k=-1)$. We show the behaviour of $x(y)$ when $\Omega_{\Lambda_{M}}<\Omega_{\Lambda_{0}}<\Omega_{\Lambda_{c}}$. The particular values for the plotting are, for Kantowski-Sachs $\Omega_{M_{0}}=2$ and $\Omega_{\Lambda_{0}}=1.5$; for Bainchi type-I $\Omega_{M_{0}}=0.5$ and $\Omega_{\Lambda_{0}}=0.5$; for Bianch type-III $\Omega_{M_{0}}=0.2$ and $\Omega_{\Lambda_{0}}=0.4$.

The behavior for the Kantowski-Sachs and Bianchi type-III cases depends on the $\Omega_{\Lambda_{0}}$ value. If $\Omega_{\Lambda_{0}} \leq \Omega_{\Lambda_{M}}$, there will be a maximum value for $y,\left(y_{m}\right)$, and since then $\dot{y}\left(y_{m}\right)=0$, the slope of the curve $x=x(y)$ will be infinite at that point. Specifically, we have $\dot{x}\left(y_{m}\right)=+\infty$ for Kantowski-Sachs, and $\dot{x}\left(y_{m}\right)=-\infty$ for Bianchi type-III, even though $x\left(y_{m}\right)$ is finite. When $\Omega_{\Lambda_{M}}<\Omega_{\Lambda_{0}}<\Omega_{\Lambda_{c}}$, after $x=y=1$ is reached we find an almost linear 
relation between the two scale factors $x$ and $y$ for the two models. While for Bianchi type-I model we have $x=y$ for the present restrictions. So, we see that for the KS model, the scale factor $a(t)$ starts from infinity if $b(t)$ starts from zero. For the Bianchi type-I model, the scale factors are always proportional or even equal. In this situation we don't have an anisotropic model; in fact, we can easily prove that this model is isotropic by a properly reparametrization of the coordinates. For the Bianchi type-III model, the scale factor $b(t)$ never starts from zero, but has an initial value different from zero when $a$ is null. The following plot shows the zones in the 2-dimensional parameter space $\left(\Omega_{M_{0}}, \Omega_{\Lambda_{0}}\right)$ where each model is allowed (Figure 3).

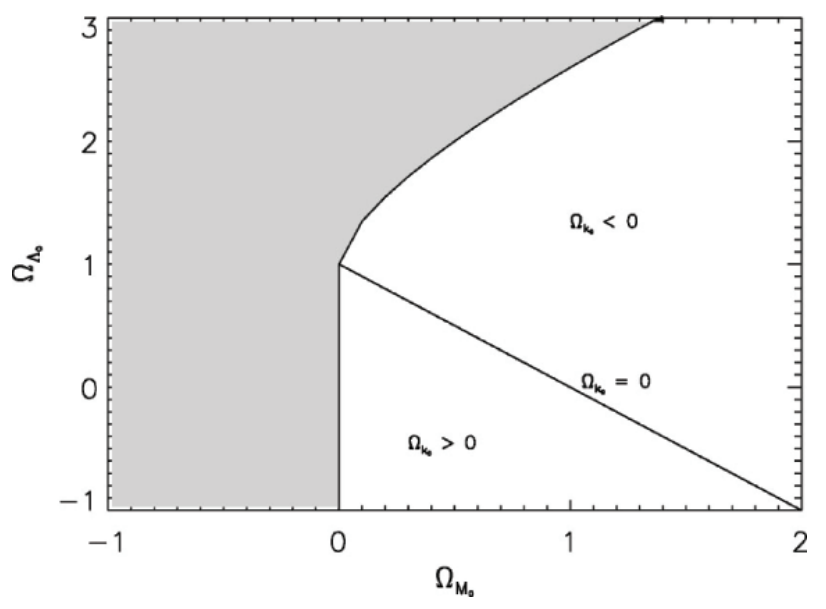

Figure 3. The Kantowski-Sachs model corresponds to the region above the straight line $\left(\Omega_{k_{0}}<0\right)$; the Bianchi type-III model corresponds the region below the straight line; $\left(\Omega_{k_{0}}>0\right)$; the straight line represents the region for the Bianchi type-I model $\left(\Omega_{k_{0}}=0\right)$. The grey zone is physically forbidden because do not reproduce the actual situation of the Universe. This zone is delimited by the equations $x=0,(24)$ and (25).

Taking into account the analysis given in [14], we may easily derive the qualitative behavior of $y(t)$, since our equation (12) is mathematically equivalent to his equation (3). Now, going back to Figure 1 and Figure 2, one can then determine the $x(t)$. The plotting below summarizes the several possibilities for the three models: Kantowski-Sachs, Bianchi type-I and Bianchi type-III models, respectively.

The present technology allows us to "see" the epoch of last scattering of radiation at a redshift of about 1000 , i.e., we can actually observe the most distant information that the Universe provides. The high level of isotropy observed from the Cosmic Microwave Background Radiation (CMBR) [15] from this epoch to our days imposes that the two Hubble factors $H_{a}$ and $H_{b}$ must remain approximately equal from this epoch to the present. In other words, we must impose a high isotropy level from the last scattering onwards, in our expressions, i.e.

$$
\frac{\Delta H}{H_{a}} \equiv \frac{H_{a}-H_{b}}{H_{a}}
$$

such that $\left|\frac{\Delta H}{H_{a}}\right| \ll 1$. From COBE 4-Year data [16], [17], we have $\left(\frac{\sigma}{H}\right)_{0} \sim 10^{-9}$ and for last scattering epoch $\left(\frac{\sigma}{H}\right)_{l s} \sim 10^{-6}$. At the last scattering we may still consider $H_{a} \simeq H_{b} \simeq H$ ( $H$ defined above $)$.
We computed several numerical integrations, with equation (27), by the following way: we gave values to $\Omega_{M_{0}}$ and $\Omega_{\Lambda_{0}}$ and integrated back in time, from now to the last scattering epoch. These $\Omega_{M_{0}}$ and $\Omega_{\Lambda_{0}}$ values were chosen such that, at last scattering epoch we had $\left|\frac{\Delta H}{H}\right|_{l s} \equiv\left|1-\left(\frac{d y}{d x}\right)_{l s}\right|=1.7 \times 10^{-6}$ or $\left(\frac{d x}{d y}\right)_{l s}=$ $1 \pm 1.7 \times 10^{-6}$. To make this we implemented a 8 order Runge-Kutta method [18].

We concluded that the sum $\Omega_{M_{0}}+\Omega_{\Lambda_{0}}$ must be close to the unity from above for Kantowski-Sachs and from below for Bianchi type-III models ${ }^{1}$. We summarized in the table below the result of imposing $\left|\frac{\Delta H}{H}\right|_{l s} \sim 1.7 \times$ $10^{-6}$ for Kantowski-Sachs and Bianchi type-III models, supposing $H_{a_{0}}=H_{b_{0}}$ (because $\left(\frac{\sigma}{H}\right)_{0} \sim 10^{-9}$ ).

From the table above we concluded that all combinations of $\Omega_{0}+\Omega_{\Lambda_{0}}$ near the unity are equally acceptable for reproducing a small anisotropy $\left((\sigma / H)_{l s} \sim 10^{-6}\right)$ at the last scattering. Nevertheless we paid special attention to the values of $\Omega_{0} \sim 0.3$ and $\Omega_{\Lambda_{0}} \sim 0.7$, since they reproduce the better fit to recent observations [19]. We have in this scenario $|\Delta H / H|<2 \times 10^{-6}$ for Kantowski-Sachs and Bianchi type-III universes. All these models approach locally an exponential expanding state $[20]$ provided the cosmological constant if we consider $\Omega_{\Lambda}>\Omega_{\Lambda_{M}}$.

\section{Conclusions}

For the Kantowski-Sachs model $\left(\Omega_{k_{0}}<0\right)$ (see Figures 4 and 5 ), we conclude that if the scale factor $b(t)$ starts from zero, then the scale factor $a(t)$ will start from infinity and decreases afterwards. When $\Omega_{\Lambda_{0}}<\Omega_{\Lambda_{M}}, b(t)$ reaches the maximum value recollapsing after that. So, $a(t)$ will reach a relative maximum, when $b(t)$ is maximum, (see Figure 4). After that, when $b(t)=0, a(t)$ goes to infinity again. When $\Omega_{\Lambda_{M}}<\Omega_{\Lambda_{0}}<\Omega_{\Lambda_{c}}$, the scale factor $b(t)$ grows indefinitely, giving place to an inflationary scenario. Then, $a(t)$ decreases reaching a minimum value, and growing after that indefinitely, and becoming proportional to $b(t)$ (see Figure 5). The initial singularity is of a "cigar" type.

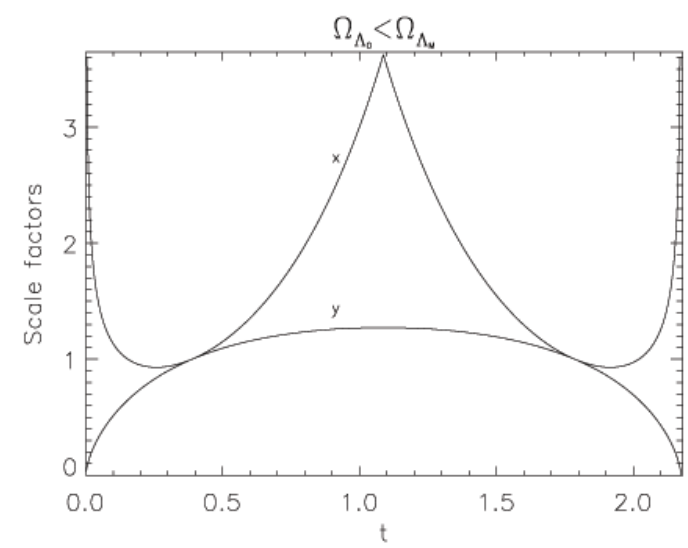

Figure 4. The scale factors $x$ and $y$ for the Kantowski-Sachs model $\left(\Omega_{k_{0}}<0\right)$ when $\Omega_{\Lambda_{0}}<\Omega_{\Lambda_{M}}$. For the plotting we put $\Omega_{M_{0}}=9$ and $\Omega_{\Lambda_{0}}=1.5$.

\footnotetext{
${ }^{1}$ It is obvious that for Bianchi type-I model $\left(\Omega_{M_{0}}+\Omega_{\Lambda_{0}}=1\right)$, with our restrictions, we have always $\Delta H / H_{a}=0$.
} 
Table 1. Density parameters and relative difference between $H_{a}$ and $H_{b}$ for Kantowsky-Sachs and Bianchi type-III models.

\begin{tabular}{ccc|ccc}
\hline & $\Omega_{M_{0}}$ & $\Omega_{\Lambda_{0}}$ & $\Omega_{0}+\Omega_{\Lambda_{0}}$ & $\Omega_{k_{0}}$ & $\frac{\Delta H}{H_{a}}$ \\
\hline K-S & 1 & $\lesssim 1.7 \times 10^{-8}$ & $1+5.6 \times 10^{-9}$ & $-1.7 \times 10^{-8}$ & $-1.6 \times 10^{-6}$ \\
K-S & $\lesssim 2 \times 10^{-15}$ & 1 & $1+6.7 \times 10^{-16}$ & $-2.0 \times 10^{-15}$ & $-1.4 \times 10^{-6}$ \\
K-S & $\lesssim 0.3+7.0 \times 10^{-9}$ & 0.7 & $1+2.3 \times 10^{-9}$ & $-7.0 \times 10^{-9}$ & $-1.7 \times 10^{-6}$ \\
K-S & 0.3 & $\lesssim 0.6+7.0 \times 10^{-9}$ & $1+2.3 \times 10^{-9}$ & $-7.0 \times 10^{-9}$ & $-1.7 \times 10^{-6}$ \\
B III & $1-10^{-10}$ & $\gtrsim 9.9 \times 10^{-11}$ & $1-3.3 \times 10^{-13}$ & $+1.0 \times 10^{-12}$ & $+1.3 \times 10^{-6}$ \\
B III & $\gtrsim 9.8 \times 10^{-14}$ & $1-10^{-13}$ & $1-6.7 \times 10^{-16}$ & $+2.0 \times 10^{-15}$ & $+1.3 \times 10^{-6}$ \\
B III & $\gtrsim 0.3-10^{-11}$ & 0.7 & $1-3.3 \times 10^{-12}$ & $+1.0 \times 10^{-11}$ & $+1.8 \times 10^{-6}$ \\
B III & 0.3 & $\gtrsim 0.7-10^{-11}$ & $1-3.3 \times 10^{-12}$ & $+1.0 \times 10^{-11}$ & $+1.8 \times 10^{-6}$ \\
\hline \hline
\end{tabular}

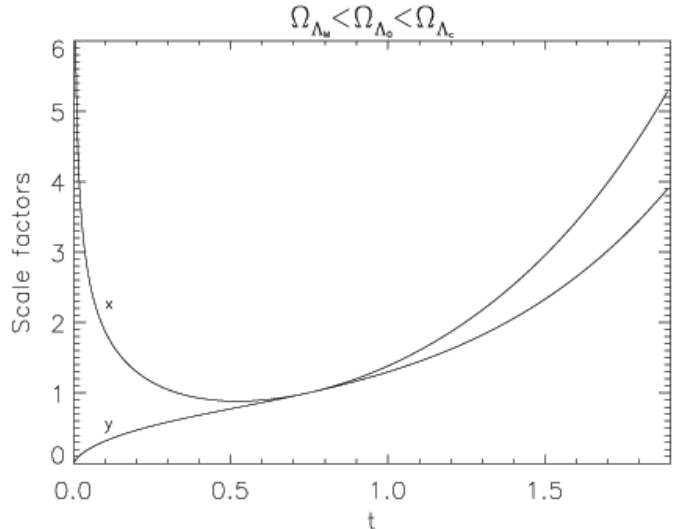

Figure 5. The scale factors $x$ and $y$ for the Kantowski-Sachs model $\left(\Omega_{k_{0}}<0\right)$ when $\Omega_{\Lambda_{M}}<\Omega_{\Lambda_{0}}<\Omega_{\Lambda_{c}}$. For the plotting we put $\Omega_{M_{0}}=2$ and $\Omega_{\Lambda_{0}}=2$.

For the Bianchi type-I model $\left(\Omega_{k_{0}}=0\right)$ (see Figures 6 and 7$)$, the scale factors $a(t)$ and $b(t)$ are proportional or even equal. Thus, this model turns out to be an isotropic one (owing to our restrictions) and $\Omega_{0}+\Omega_{\Lambda_{0}}=1$. However, when $\Omega_{\Lambda_{0}}<\Omega_{\Lambda_{M}}, a(t)$ and $b(t)$ reach the maximum and recollapse after that. And when $\Omega_{\Lambda_{M}}<\Omega_{\Lambda_{0}}<\Omega_{\Lambda_{c}}, a(t)$ and $b(t)$ grow indefinitely after an inflection.

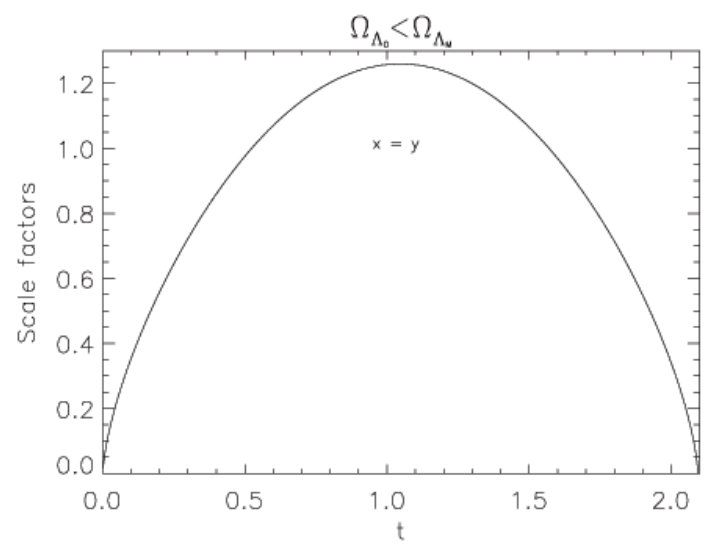

Figure 6. The scale factors $x$ and $y$ for the Bianchi type-I model $\left(\Omega_{k_{0}}=0\right)$ when $\Omega_{\Lambda_{0}}<0$. For the plotting we put $\Omega_{M_{0}}=2$ and $\Omega_{\Lambda_{0}}=-1$.

For the Bianchi type-III model $\left(\Omega_{k_{0}}>0\right)$ (see Figures 8 and 9), when $\Omega_{\Lambda_{0}}<\Omega_{\Lambda_{M}}, b(t)$ starts from an initial non vanishing value $\left(b(t=0)=b_{0}>0\right)$, reaching a maximum and recollapsing after that until reaches the same value for $t=0$. Also, $a(t)$ has a similar behavior, but starts from zero and recollapses to zero,nevertheless,

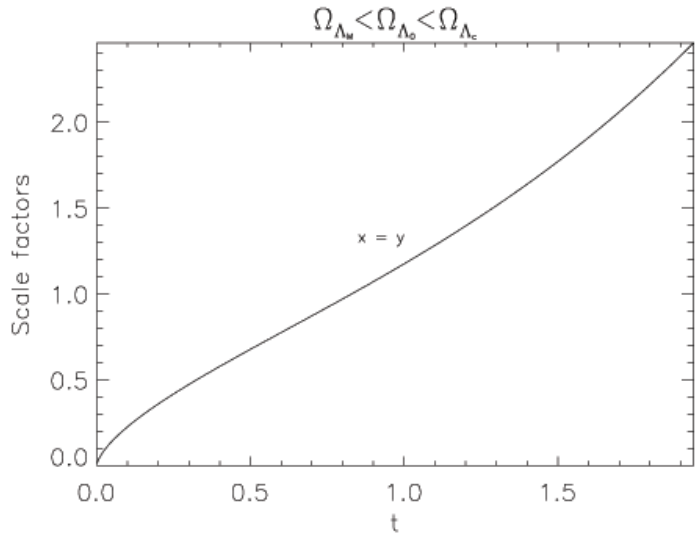

Figure 7. The scale factors $x$ and $y$ for Bianchi type-I model $\left(\Omega_{k_{0}}=0\right)$ when $\Omega_{\Lambda_{0}} \geq 0$. For the plotting we put $\Omega_{M_{0}}=0.5$ and $\Omega_{\Lambda_{0}}=0.5$.

$a(t)$ exhibits a relative minimum when $b(t)$ is maximum. When $\Omega_{\Lambda_{M}}<\Omega_{\Lambda_{0}}<\Omega_{\Lambda_{c}}, b(t)$ starts again from a non vanishing value $\left(b_{0}>0\right)$, growing indefinitely with an inflection. In this case, $a(t)$ starts from zero and grows indefinitely becoming approximately proportional to $b(t)$. So, the initial singularity is of a "pancake" type.

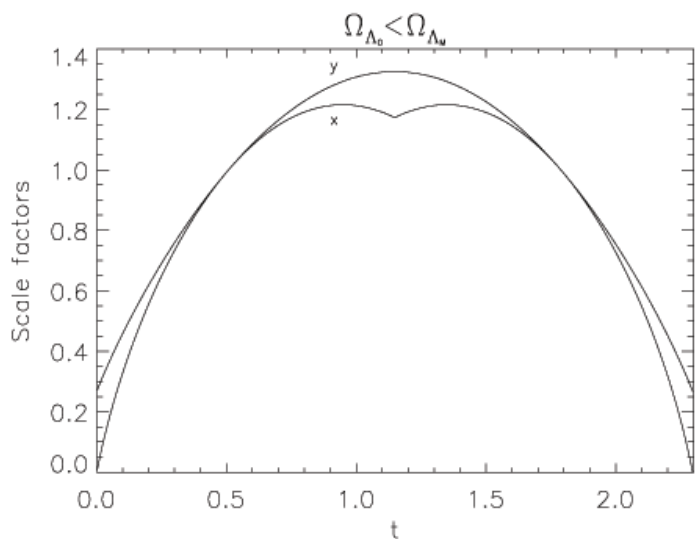

Figure 8. The scale factors $x$ and $y$ for the Bianchi type-III model $\left(\Omega_{k_{0}}>0\right)$ when $\Omega_{\Lambda_{0}}<\Omega_{\Lambda_{M}}$. For the plotting we put $\Omega_{M_{0}}=1$ and $\Omega_{\Lambda_{0}}=-1$.

In conclusion, these models undergo isotropization becoming an asymptotically FLRW, except for the Kantowski-Sachs model $\left(\Omega_{k_{0}}<0\right)$ with $\Omega_{\Lambda_{0}}<\Omega_{\Lambda_{M}}$ and for the Bianchi type-III $\left(\Omega_{k_{0}}>\right)$ with $\Omega_{\Lambda_{0}}<\Omega_{\Lambda_{M_{0}}}$. Taking into account the accuracy of the measurements of anisotropy on one hand and the fact that we can always adjust the density parameters such that $\left|\Omega_{0}+\Omega_{\Lambda_{0}}-1\right|=$ $\delta$, with $\delta \sim 10^{-9}$ on the other, we conclude that these 


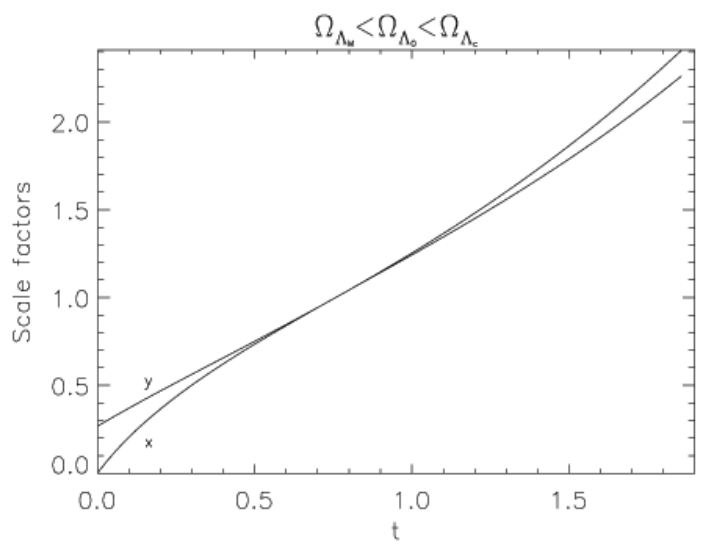

Figure 9. The scale factors $x$ and $y$ for the Bianchi type-III model $\left(\Omega_{k_{0}}>0\right)$ when $\Omega_{\Lambda_{M}}<\Omega_{\Lambda_{0}}<\Omega_{\Lambda_{c}}$. For the plotting we put $\Omega_{M_{0}}=0.2$ and $\Omega_{\Lambda_{0}}=0.4$.

models can still stand as good candidates to describe the observed Universe.

\section{REFERENCES}

[1] J. D. North, Measure Universe, (Clarendon, Oxford, 1965)

[2] V. Petrosian, Confrontation of Cosmological Theories with Obsvertational Data, (M.S. Longair, ed., Reidel, Dordrecht, 1974)

[3] J. E. Gunn and B. M. Tinsley, Nature 275, 454 (1975)

[4] S. Perlmutter et al., Nature 391, 51 (1998)

[5] A. G. Riess et al., Astron. J. 116, 1009 (1998)

[6] M. Roos, and S. M. Harun-or Rashid, Astron. Astrophys. 329, L17 (1998)

[7] S. M. Carroll, W. H. Press, and E. L. Turner, ARA\&A 30, 499 (1992)
[8] S. M. Carroll, "The Cosmological Constant", astro$\mathrm{ph} / 0004075$

[9] A. B. Henriques, Ap\&SS 235, 129 (1996)

[10] C. B. Collins and S. W. Hawking, Mon. Not. R. Astron. Soc. 162, 307 (1973)

[11] José P. Mimoso and Paulo Crawford, Class. Quantum Grav. 10, 315 (1993)

[12] A. B. Burd and D. Barrow, Nucl. Phys. B308, 929 (1988)

[13] S. Byland and D. Scialom, Phys. Rev. D 57, 6065 (1998)

[14] M. Moles, Astrophys. J. 382, 369 (1991)

[15] Peter Coles and F. Lucchin, Cosmology - The Origin and Evolution of Cosmic Structure (West Sussex: John Wiley \& Sons Ltd, 1995), p. 91

[16] E. F. Bunn, P. G. Ferreira, and J. Silk, Phys. Rev. Lett. V77 N14, 2883 (1996)

[17] A. Kogut, G. Hinshaw, and A. J. Banday, Phys. Rev. D 55, 1901 (1997)

[18] Ernst Hairer, S. P. Norsett, and G. Wanner, Solving Ordinary Differential Equations I (Springer - Verlag, Second Revised Edition, 1993)

[19] Michael S. Turner, astro-ph/9904049 (1999). To be published in the proceedings of Type Ia Supernovae: Theory and Cosmology (held at the University of Chicago, 29-31 October 1998), edited by Jens Niemeyer and James Truran (Cambridge University Press, Cambridge, UK)

[20] P. Moniz, Phys. Rev. D 47, 4315 (1993) 